\title{
Maduración ósea de niños mayores de 6 años. Muñeca y mano. I Parte: Análisis cuantitativo.
}

\author{
Dr. Mauricio Canals L. ${ }^{1}$; Dr. Carlos Valenzuela Y. ${ }^{2}$; \\ Dr. Alfredo Avendaño B. ${ }^{3}$; Dra. Sonia Samith D.4 \\ Skeletal maturity of children aged 6 - 19 years. \\ Wrist and hand. Part one. Cuantitative analysis
}

The bone maturation of hand and wrist of boys and girls aged from 6 to 19 years belonging to a longitudinal follow-up study is described. They were taken from the middle, low-middie and low socioeconomic strata of the northern district of the Metropolitan Area. The evaluation was made by the Greulich and Pyle's Atlas and the Tanner and Whitehouse's TW2 method. No differences were found between this Chilean ample and that of an English study.

(Key words: bone maturation, wrist and hand).

En trabajos anteriores hemos descrito el patrón de naduración ósea de muñeca y mano de niños chilenos de 0 a 6 años y lo hemos comparado con el inglés y el estadounidense ${ }^{1-5}$ este trabajo se refiere a la maduración ósea de muñeca y mano de niños de 6 a 19 años y de las comparaciones respectivas con esos patrones extranjeros.

\section{POBLACION Y METODO}

Los indiviauos pertenecen a la muestra del segujmiento longitudiral que se tealiza en el Area Norte de Santiago, ${ }^{6}$ En 1973 se sortearon al azar cerca de 1.000 niños de los cuales unos 700 jngr esaron al seguimiento, en el que eran controtados cada 6 meses. Cada 2 años se les indicaba una radiografía de carpo que se tomaba in el Servicio de Radiólogía del Hospital Roberto del R io o del Hospital San José del Servicio de Salud Metropolitano Norte; a este exmen concurrían voluntariamente. La maduración ósea ha siojo catalogada por uno de nosotros según el Atlas de Greulich y Pyle ${ }^{4}$ y según e]

I. Médico radiółogo.

2. Médico genetista. Departamento de Biología Celular y Genética, Facultad de Medicina, Universidad de Chile.

3. Pediatra. Departamento de Pediatria. Facultad de Medicina, Universidad de Chile.

4. Médico radiólogo. Hospital Rober to del Río.

Trabajo financiado parcialmente por Proyectos DlB L. de Ch. y pot el Servicio de Salud Metropolitano Norte. método de recuento de maduración de los huesos del carpo y de la muteca de Tannes $y$ Whitehouse. 5 Descr $\mathbf{j}$ bimos las variables edad cronológica (ECR), edad según Greulich y Pyle(EGP) y los recuentos de la evaluación del metodo de Tanner $y$ Whitehouse TW20, RUS $y$ CARP 1,2,3,5 El procesamiento estadístico y de computación ha sido descrito en los trabajos anteriores. $1,2,3$ El trabajo de Tanner, Whitehouse y Marshall describe los recuentos óseos por la mediana, nuestros datos se procesan en ptomedios $y$ destiaciones típicas; nuestros intervalos etarios son bastante estrechos por lo que el escaso número hace inútil una prueba de normalidad, de allí que comparamos nuestros promedios con la mediana inglesa; esto no presenta una limitación seria ya que no pretendemos hacer una prueba de significación estadística.

\section{RESULTADOS}

La tabla 1 presenta las variables intervato etario, número muestral, promedio de edad cronológica (ECR), promedio y desviación típica de edad según Greulich y Pyle (EGP) y el promedio y la desviación tipica de TW20, RUS y CARP para las mujeres.

La tabla 2 presenta estas variables para los varones.

Mientras las mujeres se ajustaron bien a EGP con algunos retrasos no mayores que 6 meses, los varones presentaron retrasos que en general se aproximan al año o son mayores.

La tabla 3 presenta las comparaciones de los 
promedios de recuentos obtenidos en la muestra nacional $y$ las medianas de la muestra inglesa para varones y mujeres.

Las medianas de la muestra inglesa están a una fracción cercana a la décima de desviación típica de los promedios chilenos, tanto en varones como en mujeres; en las edades prepuberales y puberales los valores no muestran una tendencia, en cambio en el período postpuberal, los valores de maduración de la muestra chilena son levemente inferiores.

\section{DISCUSION}

Como adelantamos, la población del Area Norte de Santiago de estratos socioeconómicos niedio, medio-bajo y bajo está retardada en su maduración ósea de 0 a 6 años respecto de los niños de estratos altos de Cleveland (USA), desde donde se obtuvo la muestra para el Atlas de Greulich y Pyle; sin embargo esto es más marca- do en el varón; las mujeres chilenas de esta muestra se aproximan a esos patrones. Las diferencias con la población inglesa eran menores y se daba un retardo leve sólo en los varones. ${ }^{1-3}$ Esta muestra de los mismos estratos y de la misma área prácticamente no difiere de la muestra inglesa en su maduración ósea. Si la maduración ósea es un indicador de condiciones nutricionales, como hemos demostrado, ${ }^{6}$ estos niños chilenos deberían ser considerados como en 'condiciones igualitarias de nutrición que aquellas de 1a muestra inglesa. Los valores de maduración ósea yarian según las etnias y los factores nutricionales; es así que aquellos de la muestra inglesa están por debajo de los encontrados en el Japón, especialmente en el periodo puberal y postpuberal. $^{7}$

\section{RESUMEN}

Se describe la maduración ósea đe muñeca y

Tabla 1 .

Fdad cronológica $y$ maduración ósea de la muñeca y mano según Greulich y Pyle, TW20, RUS y CARP de mujetes de 6 a 20 años en seguimiento longitudinal

\begin{tabular}{|c|c|c|c|c|c|c|c|c|c|c|}
\hline \multirow{2}{*}{$\begin{array}{l}\text { Intérvalo } \\
\text { (meses) }\end{array}$} & \multirow[t]{2}{*}{$N$} & \multirow{2}{*}{$\begin{array}{l}\text { ECR } \\
(x)\end{array}$} & \multicolumn{2}{|c|}{ EGP } & \multicolumn{2}{|c|}{ TW20 } & \multicolumn{2}{|c|}{ RUS } & \multicolumn{2}{|c|}{ CARP } \\
\hline & & & (x) & (s) & $(\mathrm{x})$ & (s) & (x) & (s) & (x) & (s) \\
\hline $65-67$ & 6 & 66,0 & 66,7 & 18.1 & 429 & 75,4 & 271 & 40,8 & 449 & 106,6 \\
\hline $71-73$ & 11 & 72,3 & 66,5 & 10,9 & 450 & 43,7 & 297 & 28,6 & 450 & 66,6 \\
\hline $77-79$ & 22 & 78,0 & 72,7 & 11,0 & 483 & 41,0 & 310 & 24,9 & 498 & 65,0 \\
\hline $83-85$ & 41 & 84,0 & 77,6 & 10,3 & 499 & 60,1 & 321 & 32,6 & 525 & 95,8 \\
\hline $89-91$ & 52 & 90,0 & 83,6 & 12,2 & $\$ 44$ & 67,0 & 347 & 37,3 & 579 & 104,3 \\
\hline $95-97$ & 55 & 95,9 & 88,2 & 12,5 & 571 & 74,1 & 362 & 48,4 & 618 & 96,2 \\
\hline $101-103$ & 42 & 101,9 & 94,4 & 8,4 & 627 & 58,3 & 399 & 41,3 & 679 & 81,6 \\
\hline 107.109 & 40 & 107,9 & 100,2 & 18,3 & 635 & 90.2 & 406 & 69,5 & 691 & 103,9 \\
\hline 113.115 & 23 & 114,0 & 103,3 & 11,2 & 677 & 67.0 & 445 & 63,7 & 725 & 76,6 \\
\hline 119.121 & 41 & 120,0 & 115,2 & 15,1 & 748 & 93,0 & 492 & 82,8 & 807 & 105,6 \\
\hline $125-127$ & 22 & 125,9 & 121,2 & 14.5 & 771 & 83,0 & 522 & 91,1 & 827 & 83,4 \\
\hline 131.133 & 44 & 132.1 & 129,2 & 12,6 & 826 & 80,9 & 575 & 87,1 & 883 & 79,0 \\
\hline $137-139$ & 10 & 137.9 & 136,8 & 20,4 & 877 & 91,6 & 667 & 158,2 & 922 & 74,7 \\
\hline $143-145$ & 62 & 144,1 & 142,5 & 9,8 & 902 & 51,5 & 667 & 73,2 & 946 & 48,3 \\
\hline $149-151$ & 20 & 150,0 & 150,9 & 15,1 & 914 & 47,0 & 713 & 89,4 & 949 & 43,9 \\
\hline $155-157$ & 52 & 156,0 & 155,9 & 13,6 & 937 & 43,4 & 744 & 105,3 & 971 & 31,3 \\
\hline 161.163 & 29 & 162,0 & 160,6 & 12,6 & 948 & 49,0 & 791 & 119.2 & 974 & 42,0 \\
\hline $167 \cdot 169$ & 28 & 168,0 & 166,9 & 13,1 & 969 & 22,7 & 844 & 97.5 & 991 & 14,9 \\
\hline 173.175 & 10 & 174.4 & 172,8 & 18,1 & 978 & 34,8 & 893 & 96,6 & 990 & 31,9 \\
\hline $179-181$ & 9 & 180,4 & 182,7 & 10,0 & 988 & 15,3 & 941 & 76,9 & 996 & 6,8 \\
\hline $185-187$ & 5 & 185,4 & 192,0 & 28,1 & 994 & 14,3 & 952 & 106,4 & 1000 & 0,0 \\
\hline $191-193$ & 3 & 192,7 & 196,0 & 18,3 & 997 & 3,6 & 977 & 30,8 & 1000 & 0,0 \\
\hline $203-205$ & 6 & 204,2 & 202,0 & 4,9 & 1000 & $\mathbf{0}, 0$ & 1000 & 0,0 & 1000 & 0,0 \\
\hline $209-211$ & 4 & 210,3 & 213,0 & 6,0 & 1000 & 0,0 & 1000 & 0,0 & 1000 & 0,0 \\
\hline $215-217$ & 4 & 216,5 & 207,0 & 11,5 & 1000 & 0,0 & 1000 & 0,0 & 1000 & 0,0 \\
\hline $219-22 ?$ & 2 & 223,0 & 216,0 & 0,0 & 1000 & 0,0 & 1000 & 0,0 & 1000 & 0,0 \\
\hline
\end{tabular}


Tabla 2.

Edad cronológica y maduración ósea de la muñeca y mano según Greulich y Pyle. TW20, RUS y CARP de varones de 6 a 19 at̄os en seguimiento longitudinal

\begin{tabular}{|c|c|c|c|c|c|c|c|c|c|c|}
\hline \multirow{2}{*}{$\begin{array}{l}\text { Intérvalo } \\
\text { (meses) }\end{array}$} & \multirow[t]{2}{*}{$\mathbf{N}$} & \multirow[t]{2}{*}{ ECR } & \multicolumn{2}{|c|}{ EGP } & \multicolumn{2}{|c|}{$\mathrm{T} W 20$} & \multicolumn{2}{|c|}{ RUS } & \multicolumn{2}{|c|}{ CARP } \\
\hline & & & $(x)$ & (s) & $(\mathrm{x})$ & (s) & $(x)$ & (s) & (x) & (s) \\
\hline $65-67$ & 2 & 66,0 & 39,0 & 4,2 & 261 & 43,1 & 154 & 9,9 & 238 & 64,4 \\
\hline $71 \cdot 73$ & 12 & 72,0 & 52,9 & 12,4 & 338 & 58,2 & 183 & 24,2 & 339 & 78,7 \\
\hline 77.79 & 19 & 77,9 & 62,1 & 9,3 & 387 & 68,4 & 212 & 34,6 & 382 & 96,4 \\
\hline 83.85 & 40 & 84,0 & 64,4 & 12,6 & 405 & 66,7 & 219 & 31,3 & 409 & 101,0 \\
\hline 89.91 & 55 & 90,0 & 74,0 & 14,1 & 456 & 70,2 & 246 & 35,3 & 469 & 101,0 \\
\hline $95-97$ & 56 & 95,9 & 74,5 & 15,4 & 477 & 69.9 & 259 & 38,4 & 487 & 95,3 \\
\hline $101 \cdot 103$ & 45 & 101,9 & 86,9 & 14,4 & $\$ 18$ & 67,2 & 275 & 34,1 & 554 & 98,7 \\
\hline $107-109$ & 40 & 107,9 & 93,2 & 18,2 & 543 & 61,7 & 288 & 36,7 & 581 & 95,3 \\
\hline $113-115$ & 33 & 113,9 & 95,1 & 19,3 & 559 & 76,4 & 298 & 40,1 & 598 & 113,2 \\
\hline 119-121 & 42 & 119,9 & 107,5 & 15.6 & 594 & 60,4 & 315 & 31,1 & 646 & 94,0 \\
\hline $125-127$ & 22 & 126,0 & 117,0 & 13,2 & 633 & 67,5 & 333 & 45,4 & 705 & 95,5 \\
\hline 131.133 & 43 & 132,0 & 118,9 & 15,2 & 654 & 63,0 & 347 & 36,0 & 723 & 91,0 \\
\hline $137-139$ & 16 & 137,9 & 130,5 & 13,9 & 693 & 62,9 & 374 & 40.4 & 764 & 86,5 \\
\hline $143 \cdot 145$ & 49 & 144,0 & 131,8 & 15,1 & 714 & 75,1 & 391 & 67,4 & 789 & 85,6 \\
\hline 149.151 & 29 & 150,1 & 142,1 & 14,0 & 765 & 85,6 & 432 & 94,7 & 846 & 89,1 \\
\hline $155-157$ & 59 & 156,0 & 147,7 & 14,4 & 810 & 90,3 & 484 & 97,3 & 885 & 87,4 \\
\hline $161-163$ & 28 & 162,0 & 151,9 & 11,0 & 836 & 89,3 & 506 & 91,0 & $90 B$ & 88,3 \\
\hline $167-169$ & 36 & 168,0 & 160,2 & 13,3 & 868 & 81,9 & 559 & 120,1 & 933 & 68,4 \\
\hline 173.175 & 11 & 174,3 & 164,7 & 14,5 & 900 & 72,7 & 611 & 92,2 & 952 & 62,3 \\
\hline $179-181$ & 14 & 180,3 & 178,7 & 16,2 & 934 & 41,9 & 695 & 159.3 & 980 & 25.2 \\
\hline $185-187$ & 3 & 186,0 & 178,0 & 13,9 & 926 & 45,2 & 647 & 103,9 & 980 & 26.7 \\
\hline 197.199 & 3 & 198.7 & 176.0 & 6.9 & 950 & 14.5 & 678 & 71,7 & 997 & 5,2 \\
\hline $203-205$ & 8 & 204,6 & 197,3 & 13,8 & 972 & 43,6 & 872 & 147,6 & 988 & 33,9 \\
\hline $209-211$ & 4 & 210,5 & 208,5 & 9,0 & 993 & 15,0 & 951 & 98,5 & 1000 & 0,0 \\
\hline $215-217$ & 6 & $216, ?$ & 207,0 & 11,8 & 989 & 24,2 & 940 & 130,9 & 998 & $s, 3$ \\
\hline $218-242$ & 5 & 227,4 & 216,0 & 12,0 & 1000 & 0,0 & 1000 & $\mathbf{0 , 0}$ & 1000 & 0,0 \\
\hline
\end{tabular}

Tabla 3.

Recucntos de maduración ósea (Tanner y Whitehouse) de una muestra inglesa (mediana) y una muesira chilena (promedios)

\begin{tabular}{|c|c|c|c|c|c|c|c|c|c|}
\hline \multirow{3}{*}{$\begin{array}{c}\text { [idad } \\
\text { (rono]ógica } \\
\text { (Heses) }\end{array}$} & \multicolumn{4}{|c|}{ Recucnto, (T $y$ W } & \multicolumn{5}{|c|}{ Recuento (T y Wi } \\
\hline & \multicolumn{2}{|c|}{ Viaronex } & \multicolumn{2}{|c|}{ Muji-ges } & $\begin{array}{c}\text { l:dad } \\
\text { Cronológica }\end{array}$ & \multicolumn{2}{|c|}{ V'arones } & \multicolumn{2}{|c|}{ Hujeres } \\
\hline & Chilcness & Ingleses & Conilenas & Ingletas & (Mcst:) & C'Jilenos & I nẹlks & Chitenas & Inglews \\
\hline 72 & 341 & 355 & 456 & 450 & 156 & 810 & 317 & $93 ?$ & 953 \\
\hline 78 & 387 & 384 & 483 & 480 & 162 & 836 & 849 & 948 & 969 \\
\hline 84 & 405 & 41.5 & 499 & 510 & $16 \mathrm{~B}$ & 868 & 879 & 969 & 981 \\
\hline 90 & 456 & 447 & 544 & $54]$ & 174 & 900 & 906 & 978 & 989 \\
\hline 96 & $47 ?$ & 477 & 571 & 571 & 180 & 934 & 931 & 988 & 995 \\
\hline 102 & 515 & 507 & 627 & 608 & 196 & 926 & 952 & 994 & 997 \\
\hline 108 & 543 & 540 & 635 & 653 & 199 & 950 & 983 & $*(193) 99 ?$ & 1000 \\
\hline $1 / 4$ & $\$ 54$ & 533 & 677 & $\mathrm{~T}(\mathrm{HO}$ & 205 & 972 & 992 & $m(198)] 000$ & 1000 \\
\hline 120 & 594 & 608 & $74 B$ & 752 & 211 & 993 & 997 & $*(204), 000$ & 1000 \\
\hline 126 & 633 & 643 & 771 & 8013 & ?I? & 989 & 1000 & 1000 & 1000 \\
\hline 132 & 654 & 675 & 826 & 845 & 227 & 10000 & 1000 & $=(223) 1000$ & 1000 \\
\hline 138 & 693 & 711 & 877 & 879 & \multirow{3}{*}{\multicolumn{5}{|c|}{ * Idadis prupjas de las mujeres. }} \\
\hline 144 & 714 & $74 ?$ & 902 & 908 & & & & & \\
\hline 150 & 765 & 781 & 914 & 932 & & & & & \\
\hline
\end{tabular}


mano de niños seguidos desde los 6 a los 19 años de edad que pertenecen a los estratos socioeconómicos medio, medio-bajo y bajo del Area Metropolitana Norte de Santiago. Se utiliza en la evaluación el Atlas de Greulich y Pyle y el método TW2 de Tanner y Whitehouse. No se encontraron diferencias apreciables con la muestra inglesa.

\section{AGRADECIMIENTOS}

Al personal del Centro de Nutrición, Crecimicnto y Desarrollo y al Servicio de Radiologia de los hospitales Robes to del Río y San José.

\section{REFERENCLAS}

1. Canals M.L., Valenzuela C.Y., Vergara P.V.: Maduración ó sea de niños de 0 a 6 años. Muñeca y mano. I Parte. Análisis cuantitativo. Rev Chil Pedintr 1985: 56: 325-328.
2. Valenzuela C.Y., Canals M.L., Vergare P.V.: Maduración ósea de riños de 0 a 6 años. Muñeca y mano. II Parte. Análisis de probitos para huesos̀ aistados. Rev Chil Pediatr 1985; $56: 329-333$.

3. Canals M.L., Valenzuela C.Y., Vergara P.V.: Maduración ósea de niños de 0 a 6 años. Muñeca y mano. III. Comparación de muestras $y$ de métodos de evaluación. Rev Med Chile 1986; 114: 419.424.

4. Greulich W.W. Pyie J.J.: Radiographic atlas of skeletal development of the hand and wrist 2 nd Edition. Stanford University Press. Stanford, Californì, USA 1959.

5. Tanner J.M., Whitehouse R.H., Marshall W.A.: Assessment of skeletal maturity and prediction of adult height (TW2 method). Academic Press, London. New York. San Francisco, 1975.

6. Avendaño A., Valenzuela C.Y., Costa R., y Cols.: Estudio alimentario de escolares con alta $y$ baja estatura según maduración ósea- Arca Norte de Santiago 1974. Pediatría (Santiago_Chile) 1976; 19: $7-11$.

7. Takai S., Akiyoshi T., and Fuchigami A.: Skeletal maturity of Japanese children in Amami-Oshima Island. Ann Hum Biol. 1984; 1 1: 57 1+5?5. 